\title{
Estudo da inclinação do plano palatino em relação à base posterior do crânio em indivíduos portadores de oclusão normal
}

\author{
Cássia T. Lopes de Alcântara Gil*, Fernando Penteado Lopes da Silva**, Ademir Tadeu Ribeiro Grossi**,

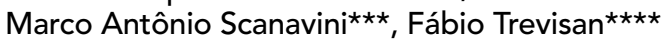

\begin{abstract}
Resumo
Objetivo: estudar a relação entre o plano palatino e a região posterior da base do crânio, em indivíduos portadores de oclusão normal. Metodologia: a amostra foi constituída por telerradiografias em norma lateral de 95 indivíduos portadores de oclusão normal natural. O plano palatino foi determinado pelos pontos Ena e Enp (Espinha Nasal Anterior e Posterior). Utilizou-se o ponto mais posterior e inferior do osso occipital (OPI), para definição da região póstero-inferior da base do crânio. Avaliou-se o comportamento do ângulo formado pelos planos OPI-Ena e Ena-Enp, denominado ângulo OPI.Ena.Enp, tendo como vértice o ponto Ena. Desta forma, valores angulares próximos a $0^{\circ}$ indicaram tendência à coincidência entre os planos OPI-Ena $\mathrm{e}$ Ena-Enp, o que equivale a dizer que, nestes casos, a extensão do plano palatino tangencia a base posterior do crânio, representada pelo Ponto OPI. Resultados: a média de valor encontrada em relação ao ângulo OPI.Ena.Enp na referida amostra foi de $-0,13^{\circ}$, valor próximo a zero, indicando tendência à coincidência entre os planos OPI-Ena e Ena-Enp. Conclusão: os resultados indicam que em pacientes portadores de oclusão normal natural, o prolongamento do plano palatino tende a tangenciar a região posterior da base do crânio, o que se revela uma característica estrutural em crânios de indivíduos portadores de oclusão equilibrada.
\end{abstract}

Palavras-chave: Plano palatino. Oclusão normal. Osso occipital. Cefalometria.

\section{INTRODUÇÃO}

A literatura sempre se preocupou com o estudo da arquitetura craniofacial do chamado "padrão normal", ou seja, oclusão equilibrada associada a uma face harmoniosa ${ }^{1,2,3,4,6,7,8,12,13}$.

Para esta finalidade geralmente são escolhidos pontos, linhas e planos de fácil localização, possi- bilitando a realização de estudos por sobreposições cefalométricas ou a obtenção de referências universais, permitindo comparações entre achados de diferentes autores.

Com este objetivo, neste trabalho estudou-se a relação entre o prolongamento do plano palatino (Ena-Enp) e a base posterior do crânio (Ponto OPI),

* Professora Titular do Programa de Pós-Graduação em Odontologia: Área de Concentração Ortodontia da Universidade Metodista de São Paulo.

** Mestrando pelo Programa de Pós Graduação em Odontologia: Area de Concentração em Ortodontia da Universidade Metodista de São Paulo. *** Coordenador do Programa de Pós-Graduação em Odontologia: Área de Concentração Ortodontia da Universidade Metodista de São Paulo.

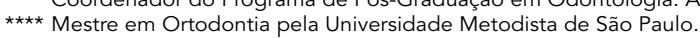


visando estabelecer um parâmetro estrutural desta região em indivíduos portadores de oclusão normal.

A escolha das referidas estruturas deve-se primeiramente ao fato de a maxila, neste caso representada pelo plano palatino, estar comumente envolvida nas discrepâncias esqueléticas em indivíduos com comprometimento facial. De igual importância é a base posterior do crânio, aqui representada pelo ponto OPI, por localizar-se posteriormente ao forame magno, pelo qual a coluna vertebral relaciona-se com o crânio.

A idéia inicial para realização do trabalho partiu dos estudos de Gil ${ }^{2,3}$, também realizados com amostras de indivíduos portadores de oclusão normal, que encontrou coincidência entre o prolongamento do plano palatino e o ponto Op (Opístio), localizado na região mais póstero-inferior do crânio, e de Sassouni ${ }^{8}$, que cita a intersecção de quatro planos horizontais, dentre eles o plano palatino, num ponto localizado posteriormente à base do crânio, denominado Centro $\underline{\mathrm{O}}$.

\section{MATERIAL E MÉTODO}

O presente estudo foi realizado no Departamento de Ortodontia da Faculdade de Odontologia da UMESP, utilizando-se telerradiografias em norma lateral de 95 indivíduos adultos portadores

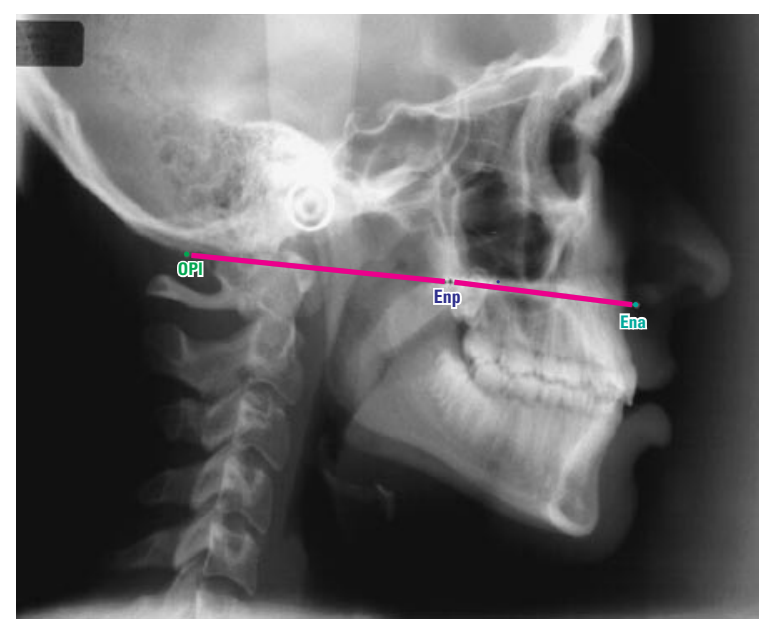

FIGURA 1 - Plano palatino (Ena-Enp) coincidente ao plano OPI-Ena, representando valor angular igual a zero. de oclusão normal natural.

Três pontos craniométricos foram utilizados para realização das medições: Ponto OPI (ponto mais póstero-inferior da base do crânio); pontos Ena (Espinha Nasal Anterior) e Enp (Espinha Nasal Posterior).

Estudou-se o comportamento do ângulo OPI. Ena.Enp, formado pelos planos OPI-Ena e EnaEnp, tendo como vértice o ponto Ena.

Para marcação dos pontos, construção dos planos e realização das medições utilizou-se o software Radiocef Studio, cedido gentilmente pela

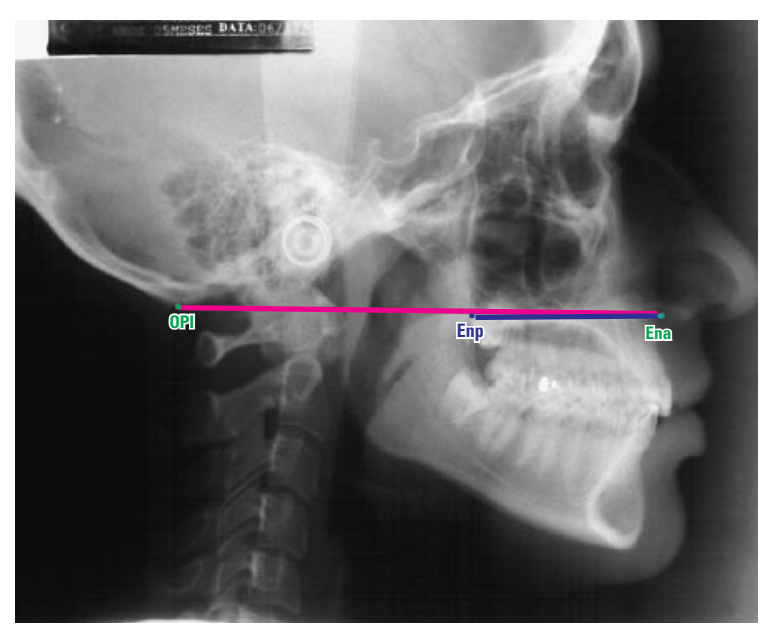

FIGURA 2 - Plano palatino abaixo do plano OPI-Ena, representando valor angular negativo.

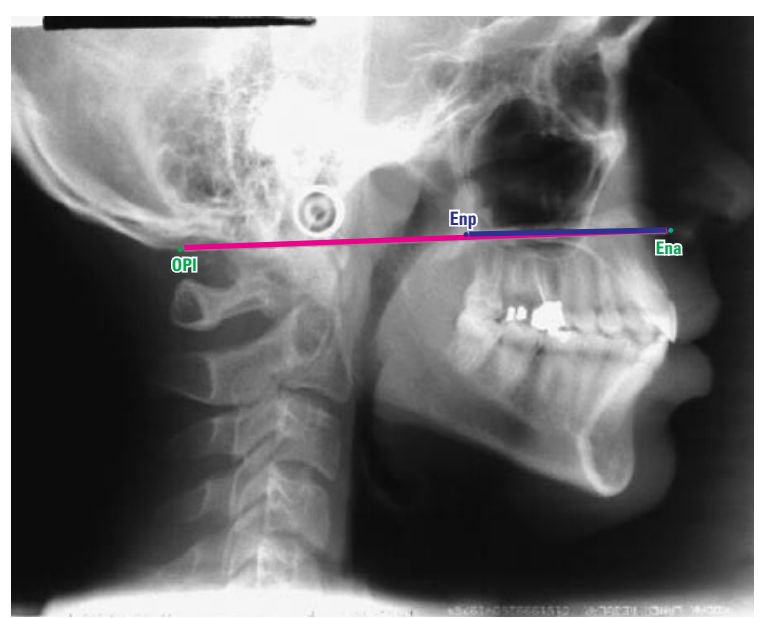

FIGURA 3 - Plano palatino acima do plano OPI-Ena, representando valor angular positivo. 
empresa Radio Memory (Belo Horizonte/MG).

Em casos onde o plano palatino passava acima do plano OPI-Ena os valores foram considerados positivos, e negativos quando o referido plano passava abaixo de OPI-Ena (Fig. 2, 3).

Mensurações angulares próximas ou iguais a zero indicaram tendência à sobreposição dos planos OPI-Ena e Ena-Enp, o que equivale a dizer que nestes casos o prolongamento do plano palatino passa pela base posterior do crânio, representada pelo ponto OPI (Fig. 1).

\section{RESULTADOS}

A obtenção das medidas referentes ao ângulo OPI.Ena.Enp foi realizada em dois tempos pelo mesmo examinador.

Desta forma, para a verificação do erro intraexaminador na obtenção das medidas angulares foram utilizados os métodos propostos por $\mathrm{Lin}^{15}$; Bland e Altman ${ }^{14}$.

A média encontrada para a diferença entre as duas medições (T1 e T2) foi de $-0,134^{\circ}$ com desvio-padrão de $\pm 0,692^{\circ}$, ou seja, a diferença entre as duas medições sempre foi inferior a $1^{\circ}$.

Após medição do ângulo OPI.Ena.Enp dos 95 indivíduos pertencentes à amostra, encontrou-se uma média de $-0,13^{\circ} \pm 1,7^{\circ}$.

Considerando a média obtida para OPI.Ena.Enp $\left(-0,13^{\circ}\right)$, seu desvio-padrão $\left( \pm 0,17^{\circ}\right)$ e a variação na sua mensuração em T1 e T2 $\left( \pm 0,692^{\circ}\right), 82,11 \%$ da amostra apresentou-se dentro da faixa de valores entre $-2,5^{\circ}$ e $2,2^{\circ}$ para o referido ângulo, sendo considerados dentro de uma distribuição normal ${ }^{9}$.

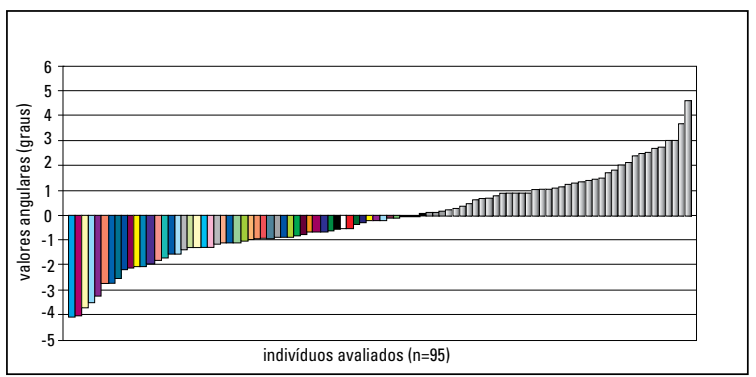

GRÁFICO 1 - Distribuição dos valores de OPI.Ena.Enp na amostra estudada.
Este resultado mostra a tendência à coincidência entre os planos OPI-Ena e Ena-Enp, demonstrando que, em indivíduos portadores de oclusão normal, o prolongamento do plano palatino tende a tangenciar a base posterior do crânio.

Avaliando-se o gráfico 1, representativo da distribuição dos valores de OPI.Ena.Enp dos 95 indivíduos da amostra estudada, nota-se um grande equilíbrio em relação aos valores positivos e negativos do referido ângulo.

O mesmo gráfico mostra de forma ilustrativa que a distribuição de valores de OPI.Ena.Enp deuse de forma homogênea, mostrando um equilíbrio entre a quantidade de indivíduos com o plano palatino acima e abaixo da linha OPI-ENA.

O gráfico 2 evidencia que a maior parte da amostra $(82,11 \%)$ encontra-se numa faixa de valores angulares entre $-2,5^{\circ}$ e $2,2^{\circ}$.

Este resultado indica que a maioria dos indivíduos portadores de oclusão normal apresenta tendência à coincidência entre o prolongamento do plano palatino e a região póstero-inferior do crânio.

Observa-se que a quantidade de indivíduos que apresentaram inclinação do plano palatino acima $(9,47 \%)$ e abaixo $(8,42 \%)$ do plano OPI-Ena apresenta-se muito equilibrada, indicando não haver prevalência de rotação do referido plano no sentido horário ou anti-horário em indivíduos portadores de oclusão normal.

\section{DISCUSSÃO}

A maior parte dos autores preocupa-se em correlacionar a mandibula com estruturas mais es-

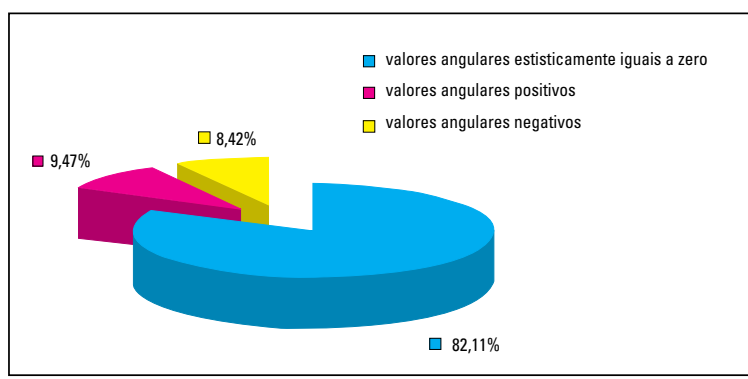

GRÁFICO 2 - Distribuição dos valores de OPI.Ena.Enp na amostra estudada. 
táveis do esqueleto facial em suas análises cefalométricas, já que em grande parte dos casos de má oclusão com comprometimento estético, a mandíbula é o principal causador do problema, como na Classe II com retrognatismo ou na Classe III com prognatismo mandibular ${ }^{7}$.

Com isto, os relatos da literatura restringem-se ao estudo do posicionamento ântero-posterior da maxila em relação à base do crânio, seja de forma angular $^{7}$ ou linear ${ }^{5}$. $\mathrm{O}$ aspecto da angulação do plano palatino em relação à estruturas mais estáveis é muitas vezes desconsiderado, o que não ocorre em relação à mandíbula, que possui maior número de medidas para esta finalidade ${ }^{7,10,11}$.

Neste trabalho os autores propuseram-se a estudar o padrão "normal" em relação à inclinação do plano palatino, já que em muitos casos esta estrutura pode apresentar-se com rotação no sentido horário ou anti-horário, acentuando respectivamente padrões braqui e dolicofaciais.

$\mathrm{Gil}^{2}$, ao estudar a presença da Proporção Áurea em indivíduos com oclusão normal, observou que quando a porção menor do compasso áureo correspondia à distância Ena-Enp, a terceira ponta do instrumento invariavelmente dirigia-se à região mais póstero-inferior do crânio. Verificou que o prolongamento do plano palatino tangenciava esta região e que tal fato deveria ser considerado ao pensar-se nas divisões naturais do crânio.

A relação entre o prolongamento posterior dos planos basal superior, palatino, oclusal e mandibular foi estudado por Sassouni ${ }^{8}$ em indivíduos com oclusão normal. $\mathrm{O}$ autor encontrou que, nestes indivíduos, pelo menos dois dos quatro planos se cruzam na região posterior ao contorno occipital, mas não houve relação entre o prolongamento dos mesmos e a região basal do crânio.

Em 82,11\% da amostra estudada, os valores de OPI.Ena.Enp encontraram-se entre $-2,5^{\circ}$ e $2,2^{\circ}$, mostrando-se, portanto, valores reduzidos e com pouca variação se comparados a outros estudos referentes à inclinação do plano palatino. Ricketts $^{6}$ encontrou em sua amostra de oclusão normal uma média de $1^{\circ} \pm 3,5^{\circ}$ e Urbano $^{12}$ de $1,03^{\circ} \pm$ $3,96^{\circ}$, ambos considerando o ângulo formado entre os planos palatino (Ena-Enp) e de Frankfurt (Po-Or).

Os achados deste estudo demonstram de forma numérica, por meio da mensuração angular de OPI.Ena.Enp, que existe a tendência do prolongamento do plano palatino tangenciar a região póstero-inferior da base craniana em indivíduos com oclusão normal.

Esta colocação torna-se de grande importância à medida que estabelece um padrão de normalidade para a inclinação do plano palatino em relação à base posterior do crânio, desconsiderada em outras análises cefalométricas.

Sem dúvida, esta característica do "normal" pode ser usada como referência para o restabelecimento da inclinação do plano palatino onde a mesma estiver alterada, em casos ortodônticos ou orto-cirúrgicos.

As principais vantagens do método empregado são:

- utilização de poucos pontos de referência (OPI, Ena e Enp);

- possibilidade de aplicação sem necessidade de medidas-padrão,

- baseada na proporção craniofacial do próprio indivíduo;

- emprego da base posterior do crânio como referência para avaliação do plano palatino, que parece-nos mais confiável em relação ao plano de Frankfurt $^{13}$, utilizado em outros estudos ${ }^{6,12}$.

\section{CONCLUSÃO}

A partir do estudo realizado, pode-se concluir que, em indivíduos com oclusão normal:

- o prolongamento posterior do plano palatino (Ena-Enp) tangencia a região mais póstero-inferior do crânio;

- o plano palatino encontra-se à mesma altura da base posterior do crânio;

- a inclinação do plano palatino entre $-2,5^{\circ}$ e $2,2^{\circ}$ pode ser usada como referência para o resta- 
belecimento da inclinação do referido plano, em casos onde a mesma estiver alterada;

- o presente estudo utilizou-se de medidas obtidas de cada indivíduo, sem a utilização de médias amostrais, o que confere individualização à análise.

\title{
Study of palatal plane inclination related to the posterior cranial base in subjects with normal occlusion
}

\begin{abstract}
Aim: to study the inclination of the palatal plane in 95 subjects with clinically normal occlusion. Methods: the study was made using lateral headfilms and the results showed that in normal occlusion the extension of the palatal plane (ANS-PNS) passes trough the most posterior and inferior region of the skull, defined as Point "OPI". Results: OPI.Ena.Enp mean value was $-0.13^{\circ}$, close to zero. Conclusion: the results indicated that in normal occlusion patients the palatal plane presents a tendency to be tangent with posterior cranial base.
\end{abstract}

Key words: Palatal plane. Normal occlusion. Occipital bone. Cephalometrics.

\section{REFERÊNCIAS}

1. ANGLE, E. H. Treatment of malocclusion of the teeth. Angle's system. 7th ed. Philadelphia: S. S White. 1907. p. 60-87; 159-160.

2. GIL, C. T. L. A. Estudo da proporção áurea na arquitetura do crânio de indivíduos com oclusão normal, a partir de telerradiografias laterais, frontais e axiais. 1999. $183 \mathrm{f}$. Tese (Doutorado em Odontologia - Área de Concentração em Radiologia Odontológica)-Faculdade de Odontologia, Campus de São José dos Campos, Universidade Estadual Paulista "Júlio de Mesquita Filho", São José dos Campos, 1999.

3. GIL, C. T. L. A. Proporção áurea craniofacial. São Paulo: Ed. Santos, 2001.

4. GIL, C. T. L. A.; MÉDICI FILHO, E. Estudo da proporção áurea na arquitetura craniofacial de indivíduos com oclusão normal, a partir de telerradiografias axiais, frontais e laterais. Ortodontia, São Paulo, v. 35, n. 2, p. 69-85, abr./jun. 2002.

5. McNAMARA JR., J. A method of cephalometric evaluation. Am J Orthod, St. Louis, v. 86, no. 6, p. 449-469, Dec. 1984.

6. RICKETTS, R. M. et al. Orthodontic diagnosis and planning: their roles in preventive and rehabilitative dentistry. [United States]: Rocky Mountain, 1989. v. 1.

7. RIEDEL, R. A. The relation of maxillary structures to cranium in malocclusion and in normal occlusion. Angle Orthod, Appleton, v. 22 , no. 3, p. 142-145, 1952.

8. SASSOUNI, V. A. A roentgenographic cephalometric analysis of cephalofacio- dental relationships. Am J Orthod, St. Louis, v. 41 no. 10, p. $735-764$, Oct. 1955.
9. SPIEGEL, M. R. Estatística. 3. ed. São Paulo: Makron Books, 1993.

10. STEINER C. C. Cephalometrics in clinical practice. Angle Orthod, Appleton, v. 29, no. 1, p. 8-29, Jan.1959.

11. TWEED, C.H. The Frankfort mandibular plane angle in orthodontic diagnosis, classification, treatment, planning and prognosis. Am J Orthod Oral Surg, St. Louis, v. 32, no. 4, p. 175-230, Apr. 1946.

12. URBANO, A. L. Avaliação cefalométrica radiográfica de jovens leucodermas com oclusão normal, segundo a análise lateral de Ricketts. 2003. 195 f. Dissertação (Mestrado em Odontologia)-Universidade Metodista de São Paulo, São Bernardo do Campo, 2003.

13. ZAPATA, M. R. S. M. Estudo cefalométrico-radiográfico da posição natural da cabeça em pacientes com oclusão normal 2003. 177 f. Dissertação (Mestrado em Odontologia)-Universidade Metodista de São Paulo, São Bernardo do Campo, 2003.

14. BLAND, J. M.; ALTMAN, D. G. Measuring agreement in method comparison studies. Stat Methods Med Res, London, v. 8, p. 135-160, 1999

15. LIN, L. I. A concordance correlation coefficient to evaluate reproducibility. Biometrics, Washington, D. C., v. 45, no.1, p. 255-268, Mar. 1989.

\section{Endereço de correspondência}

Cássia T. Lopes de Alcântara Gil

Rua Afonso Brás 525 - Vila Nova Conceição

CEP: 04.511-902- São Paulo/SP

E-mail: cassia.gil@uol.com.br 